\title{
Color screening in flux tubes and in the color Coulomb potential from the QCD Field Correlators *
}

\author{
M.S. Lukashov ${ }^{a, b, \dagger}$ and Yu.A. Simonov ${ }^{a, \ddagger}$ \\ a Alikhanov Institute for Theoretical and Experimental Physics, \\ B. Cheremushkinskya 25, 117218 Moscow, Russia \\ ${ }^{b}$ Moscow Institute of Physics and Technology, \\ Institutskiy per. 9, 141700 Dolgoprudny, Moscow Region, Russia
}

June 28, 2017

\begin{abstract}
Colorelectric and Colormagnetic structure of the flux tubes, connecting heavy quark and antiquark, is investigated in the framework of the Field Correlator method which describes all resulting fields in terms of correlators $D^{E}$ and $D_{1}^{E}$. The latter have been computed via gluelumps, which allows to predict the resulting distribution of color fields $\mathbf{E}(\mathbf{r})$, and colormagnetic currents $\mathbf{k}(\mathbf{r})$ in the flux tubes. It is shown, that at large distances $r \gg \lambda \approx 0.2 \mathrm{fm}$ the whole structure of fields and relations between them is similar to that of the dual superconductor theory, but the basic dynamics, including small distances, is given by field correlators of the real stochastic vacuum. The important contradiction between the strong screening of color fields in the width of flux tubes and almost no screening in the perturbative $Q \bar{Q}$ potential is resolved.
\end{abstract}

Keywords: color screening, flux tubes

\footnotetext{
*arXiv: v2, 28-06-17.

†lukashov@phystech.edu

¥simonov@itep.ru
} 


\section{Introduction}

The flux tubes between heavy quark and antiquark are considered as a necessary consequence of the color confinement mechanism, and were investigated numerically on the lattice during the last three decades, see e.g. [1 24.

It was understood that this physical phenomenon should exist, whatever is the mechanism of color confinement, and hence only the detailed structure of flux tube fields can distinguish between different models of confinement.

One of the most popular, however not derived from QCD, is the model of dual superconductor (MDS) [25, 26], where the QCD vacuum can be represented as a coherent state of colomagnetic monopoles. The numerous studies, both in theory and in numerical lattice works, have been done for the last two decades, trying to find the reasonable arguments and explicit formalism for MDS, see the review papers [27,28] and the references therein.

The difficulty of this approach is the lack of colormagnetic monopoles as real physical objects or Euclidean solutions of QCD, so that one should consider those as effective degrees of freedom in the real physical vacuum of QCD.

Meanwhile the quantitative theory of color confinement was suggested in [29] 31] based on vacuum field correlators, developed in detail for application in hadron properties [32,33, including theory of Regge trajectories etc., the theory of chiral symmetry breaking [34], perturbation theory [35] and the QCD thermodynamics 36].

Field correlators can be found from the solution for the gluelump Green's functions of [37 39], which are connected back to field correlators in a selfconsistent way [40 42], which allows to define their properties for distances $r>\lambda$, where $\lambda \approx 0.2 \mathrm{fm}$ is the vacuum correlation length, $\lambda \sim 1 / M_{\text {glp }}, M_{\text {glp }}$ is the lowest gluelump mass.

The problem of flux tubes in the framework of field correlator formalism was discussed in the review papers [43,44], where it was shown, that the basic properties of flux tubes are easily obtained from this formalism.

Recently a new formulation of the QCD equation of state and temperature transition was accomplished [45], which required a very detailed structure of the confinement dynamics, i.e. of the properties of field correlators, and those can be tested in the flux structure.

The latter, as shown below, define all the fields in the flux tube, and inversely, the study of flux tube fields gives information about details of field correlators, i.e. the details of the confinement mechanism.

From this point of view the flux tubes are an important source of information about the structure of confinement, including the temperature dependence of its constructing details.

Recently new lattice measurements of flux tube structure have been done in [46 48] and specifically in [46] the first accurate results have been obtained both for $S U(3)$ and $2+1$ QCD. It is the purpose of the present paper to compare these results with our analytic approach and to draw some conclusions on the mechanism of flux tubes and confinement.

The extended study of flux tubes in the framework of FCM was done in [44], where the CE field distribution $\mathbf{E}(\mathbf{r})$ was found in terms of the confining correlator $D^{E}(x)$ and perturbative correlator $D_{1}^{E}(x)$, yielding the standard picture of the QCD string between two sources $Q$ and $\bar{Q}$. In addition an important step was done, defining the colormagnetic current $\mathbf{k}(\mathbf{r})$, which has the form of rings around the string, and it was also shown, that 
the "dual" London equation rot $\mathbf{k}=\lambda^{-2} \mathbf{E}(r)$ - is satisfied at large distances from the string axis, $r \gg \lambda$.

This fact actually supports the idea, that the Field Correlator theory of confinement at large distances to some extent is equivalent to the dual superconductor picture, however the former allows to describe the flux tube fields at all distances.

It is one of the aims of our paper, to go further in this direction and in particular to present the distribution $|\mathbf{k}(\mathbf{r})|$ as a function of $r$, which can be further computed numerically on the lattice.

In addition, an interesting consequence of our theory is the distribution of the CE field $\mathbf{E}$, which is produced by the color charges and screened in the transverse, but not longitudinal direction as described by the correlator $D_{1}^{E}$ and has no equivalent in MDS.

Indeed, the FC describe two kinds of CE fields, $E^{(1)}$ and $E^{(D)}$ due to correlators $D^{E}$ and $D_{1}^{E}$ respectively, and they have completely different distributions, in particular, $D^{E}$ gives the main body of the flux tube,while $D_{1}$ gives the screening of the color Coulomb interaction.

Actually in [44, the important problem of the screening of perturbative fields due to confinement was not fully investigated, and instead there was a requirement of this screening at large distances, $r \gg \lambda$, where $\lambda \approx 0.2 \mathrm{fm}$ is the vacuum correlation length. Below we give the full answer to this problem of screening, based on the theory of confinement.

Another important development of the analysis of flux tube is its temperature variation, which was done on the lattice in [49]. This allows to measure the $T$ dependence of correlators $D^{E}, D_{1}^{E}$, which plays the crucial role in the temperature transition region, as shown in [45].

The paper is organized as follows. In the next section we list the basic definitions and equations of the FCM, related to flux tubes, and in the section 3 define the fields inside flux tubes in terms of FC, and magnetic currents. In section 4 our results are shown and compared to existing data for $T=0$. Discussion of results and prospectives are given in the concluding section.

\section{Field correlators in QCD}

The vacuum fields $F_{\mu \nu}(x)$ in QCD without external currents are necessarily stochastic and can be characterized by the set of Field Correlators (FC), which in the gauge invariant form for the lowest one, the Gaussian, 29 31 can be written as

$$
\begin{aligned}
g^{2} D_{i 4 k 4}^{(2)}(x-y) & \equiv \frac{g^{2}}{N_{c}}\left\langle\operatorname{tr}_{f}\left(F_{i 4}(x) \Phi(x, y) F_{k 4}(y) \Phi(y, x)\right\rangle=\left(\delta_{i k}\right) D^{E}(x-y)+\right. \\
+ & \frac{1}{2}\left(\frac{\partial}{\partial x_{i}}\left[h_{k}+\text { perm }\right]\right) D_{1}^{E}(x-y), \quad h_{\lambda}=x_{\lambda}-y_{x} .
\end{aligned}
$$

The temporal Wilson loop in terms of this basic FC can be written as [29] 31 via colorelectric FC, $D^{E}$ and $D_{1}^{E}$

$$
\begin{gathered}
W(C)=\frac{1}{N_{c}}\left\langle\operatorname{tr} P \exp \left(i g \int_{C} d z_{\mu} A_{\mu}(z)\right)\right\rangle= \\
\frac{1}{N_{c}}\left\langle\operatorname{tr} P \exp \left(i g \int_{S_{\min }} d \sigma_{\mu \nu} F_{\mu \nu}\right)\right\rangle=\frac{1}{N} \operatorname{tr} P \exp \left[-\frac{g^{2}}{2} \int d \sigma_{\mu \nu} d \sigma_{\lambda \rho}\left\langle F_{\mu \nu} F_{\lambda \rho}\right\rangle+\ldots\right] \cong
\end{gathered}
$$




$$
\cong \exp \left(-S_{\min } \frac{1}{2} \int D^{E}(z) d^{2} z\right)
$$

which implies that the string tension is expressed via $D^{E}(z)$ as

$$
\sigma^{E}=\frac{1}{2} \int d^{2} z D^{E}(z)
$$

In (3) the integration is over the minimal surface $S_{\text {min }}$ inside the Wilson loop $C$.

Using $D^{E}$ and $D_{1}^{E}$ one can define the instantaneous interaction between fundamental or adjoint color changes, as it shown in the Appendix 1.

Note that $D^{E}(x)$, which enters in (2), generates the scalar potential $V_{D}(r)$

$$
V_{D}(r)=2 c_{a} \int_{0}^{r}(r-\lambda) d \lambda \int_{0}^{\infty} d \nu D^{E}(\lambda, \nu)=V_{D}^{(\operatorname{lin})}(r)+V_{D}^{\mathrm{sat}}(r) .
$$

The FC $D_{1}^{E}$, which enters in the full derivative in (1), creates the vector-like interaction

$$
V_{1}(r)=c_{a} \int_{0}^{r} \lambda d \lambda \int_{0}^{\infty} d \nu D_{1}^{E}(\lambda, \nu), \quad c_{\text {fund }}=1, \quad c_{\text {adj }}=\frac{9}{4} .
$$

Eqs. (44) and (5) yield the information on correlators $D^{E}, D_{1}^{E}$, which can be obtained from the study of $Q \bar{Q}$ potentials $V_{D}(r), V_{1}(r)$. In what follows we shall exploit another way: on one side we shall define $D^{E}$ and $D_{1}^{E}$ via gluelump Green's function, on another side we find the structure of flux tubes with the help of $D^{E}, D_{1}^{E}$. In this way the data on flux tubes can be predicted and compared with lattice or experimental sources.

Till now the properties of $D^{E}$ and $D_{1}^{E}$ were not defined and to get information on that, one should exploit their connection to the gluelump Green's function, as it was done in [40 42]. Namely, $D^{E}(x)$ is expressed via the two-gluon-gluelump Green's function $G^{(2 g)}(x, y)$

$$
D^{E}(x-y)=\frac{g^{4}\left(N_{c}^{2}-1\right)}{2} G^{(2 g)}(x, y) .
$$

The lowest eigenvalues and the asymptotics of $G^{(2 g)}$ were found in [37], namely $M_{0}^{(2 g l)} \approx$ $2.5 \mathrm{GeV}$, and from 40,42

$$
G^{(2 g)}(x)\left(x \gg 1 / M_{0}^{(2 g l)}\right) \approx 0.108 \sigma_{f}^{2} e^{-M_{0}^{(2 g l)}|x|} .
$$

The insertion of (7), (6) into (4) immediately yields the potential $V_{D}^{(\text {lin) }}(r)$ which is linear in whole region $r>1 / M_{0}^{(2 g)} \approx 0.1 \mathrm{fm}$. This fact agrees well with all experimental and numerical data.

It is interesting, that the same approach of two-gluon gluelump asymptotics for the colormagnetic function $D^{H}(x-y)$ yields [50] the well-known relation, found also on the lattice [51]

$$
\sigma^{H}=g^{4}\left(N_{c}^{2}-1\right) T^{2} c_{\sigma}, \quad c_{\sigma}=\text { const. }
$$

In contrast to that, the FC $D_{1}^{E}$ is expressed via the one-gluon gluelump Green's function with the nonperturbative part, behaving asymptotically as 4042

$$
D_{1}^{E(\text { nonp })}(x)=\frac{2 N_{c} \alpha_{s}}{x} M_{0}^{(1 g l)} \sigma_{f} e^{-M_{0}^{(1 g l)}|x|}
$$


where $M_{0}^{(1 g l)} \cong 1.5 \mathrm{GeV}$ [37 39], while the total form, containing the perturbative part as shown in Appendix 2, is

$$
D_{1}^{E(\text { pert })}(x)=\frac{2\left(N_{c}^{2}-1\right) \alpha_{s} K_{2}(m x)}{N_{c} \pi x^{2}}+O\left(\alpha_{s}^{2}\right) .
$$

As a result, the FC $D_{1}^{E}$ produces the interaction $V_{1}^{E}(r)$

$$
V_{1}^{E}(r)=-\frac{\left(N_{c}^{2}-1\right) \alpha_{s} e^{-m r}}{2 N_{c} r}+O\left(\alpha_{s}^{2}\right), \text { where } m=O(1 \mathrm{GeV}) .
$$

One should stress at this point, that one-gluon and two-gluon gluelumps enter separately in their mass measurements both on the lattice [38, 39] and analytically [37], yielding different values $(2.5 \mathrm{GeV}$ in (77) and $1.5 \mathrm{GeV}$ in (9) in both approaches. On the other hand, when computing the $\mathrm{FC}$ via gluelumps, as in [40 42], the mixing term $\operatorname{appears}\left(D_{\mu \nu, \lambda \sigma}^{(1)}\right.$ in [40 42]) which mixes up two contributions and brings in an averaged value $\bar{M}$ of the order of $1.0 \mathrm{GeV}$. The resulting mass $m$ of the order of $\bar{M}$ in Eq.(11) is the gluon screening mass.

At this point we turn to the measurements the flux tube fields, as it is done on the lattice, where one computes the average value of the contour, shown in Fig.1, consisting of a small plaquette at the point $x$, connected by two fundamental lines to the Wilson loop of heavy quarks $Q, \bar{Q}$.

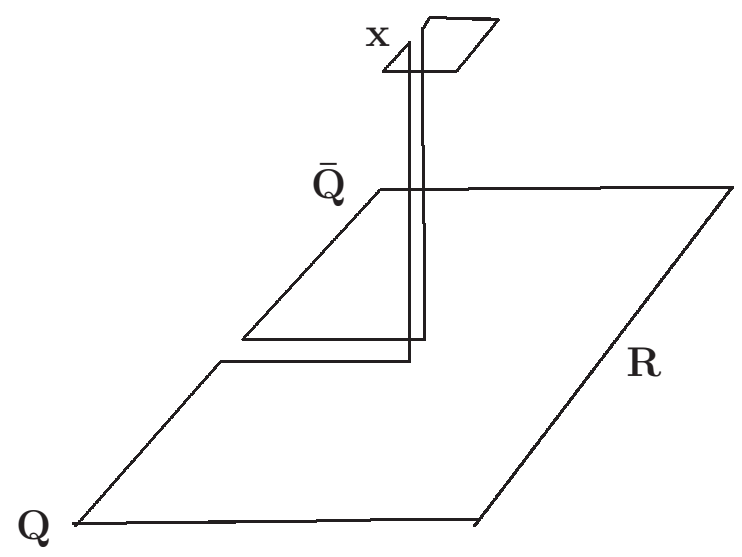

Figure 1: The connected probe plaquette at the point $x$ above the $Q \bar{Q}$ Wilson loop.

Here appears a new phenomenon, which might be called "the quenching of the screening gluon mass", namely, as shown in Fig.2, the value of the one-gluon screening mass $M \cong 1.5 \mathrm{GeV}$ is obtained, when the parallel transporter in the transverse position is fixed, as shown in Fig. 1, where the double fundamental line in the transverse direction defines the form of the confining film in the gluelump Green's function.

On the other hand, measuring gluon exchange in the horizontal plane without any transporters, which limit the size of the confining film, one obtains, that the resulting minimal surface is a slightly deformed plane inside the big contour, as shown in Fig. 3. The energy of deformation is equal to $\varepsilon_{\text {plane }}=\sigma \Delta S_{\min }=\sigma \frac{h^{2}}{L}$, where $h$ is the average deflection of gluon path from the plane and $L$ is plane length. This should be compared 


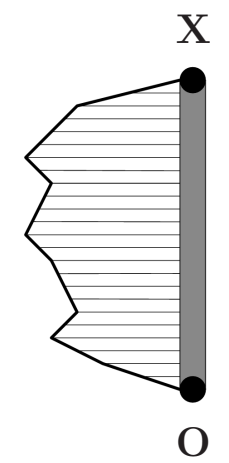

Figure 2: The $1 \mathrm{~g}$ gluelump configuration for the transverse probe.

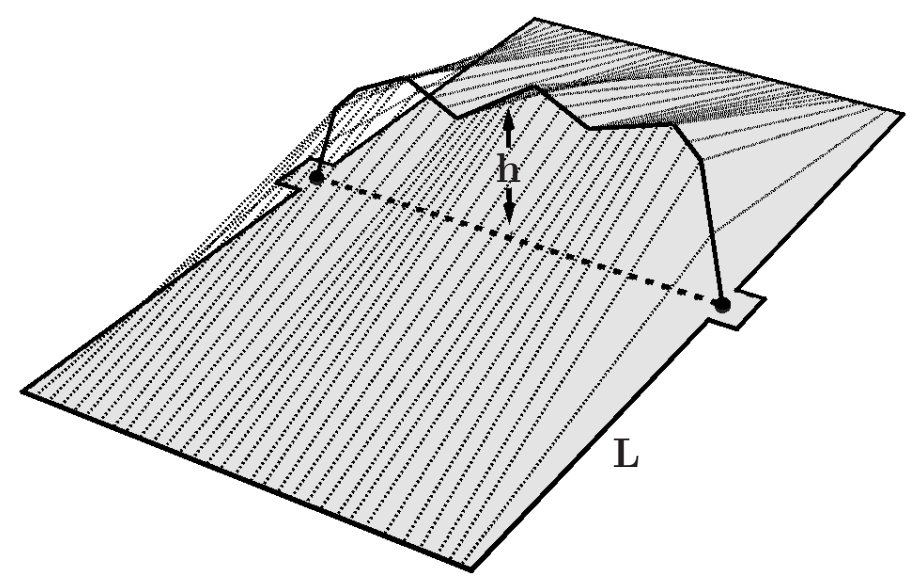

Figure 3: The minimal area surface for the gluon exchange interaction.

with the energy in the gluelump case, $\varepsilon_{g l p} \cong \sigma h$, with the result $\varepsilon_{\text {plane }} \ll \varepsilon_{\text {glp }}$, meaning a strong damping of the Coulomb screening $M_{s c r}^{C o u l} \ll M_{g l p}$.

Moreover, the length $L$ between consequitive gluon exchanges can be estimated from the action exponent $\exp \left(-V_{\text {Coul }}(R) L\right)$, as $L^{-1} \sim V_{\text {Coul }}(R) \sim \frac{4}{3} \frac{\alpha_{s}}{R}$, leading to the result $\varepsilon_{\text {plane }}=\sigma \frac{h^{2}}{L} \sim \frac{4}{3} \sigma \frac{h^{2}}{R} \alpha_{s}$. Thus the screening is additionally damped at large $R$, for the analytic treatment of this type of interaction (but without $\alpha_{s} / R$ ) see [52].

It is clear from the analysis of [52], that the screening mass, corresponding to the Fig. 3, is the mass excitation of the static hybrid of the length $L$ with the transverse excitation of the order of $\sqrt{12} / L$ and longitudinal $(\sigma / L)^{1 / 3}$. Defining $L$ as before from the condition $\Delta t V_{\text {Coul }}=O(1)$, one obtains for $R=1 \mathrm{fm}$ for both excitations $\Delta m \approx 0.4$ $\mathrm{GeV}$ instead of $1.4 \mathrm{GeV}$ for the one-gluon gluelump mass. Note also, that for the single gluon exchange, when $\Delta t$ tends to infifnity, the screening mass $\Delta m$ tends to zero.

Numerically on the lattice the static $Q \bar{Q}$ potential demonstrates [53, 54] the linear plus pure Coulomb form. Our discussion above might give an answer to the similar point, raised recently in [55].

In our case, where a part of parallel transporters is in the transverse direction, as in Fig. 1, one expects, that our mass $m$ satisfies $M_{s c r}^{\text {Coul }}<m<M_{g l p}$, and we choose explicitly in what follows for transverse $r_{\perp}$ distributions, $m=1 \mathrm{GeV}$, which we shall use 
to define the Vacuum Correlation Length $\lambda$,

$$
\lambda=\frac{1}{m}=0.2 \mathrm{fm}
$$

To complete the picture of potentials $V_{D}$ and $V_{1}$ one should mention another important feature of resulting potentials in (44), (11): as it is shown in the appendix of [45], the terms $V_{D}^{\text {sat }}(r)$ in (4I) and $V_{1}^{(n p)}(r)$ have different signs and almost fully compensate each other for low temperatures. As a result the interaction between two static charges acquires the well established form, confirmed on the lattice and in experiment:

$$
V_{Q \bar{Q}}(r)=V_{1}^{(\text {pert })}(r)+V_{D}^{(\text {lin })}(r) .
$$

As we shall see below, this cancellation holds only for potentials, which are in-plane integrals of the FC, as in (4), (5). However, for the flux tube probes, which are mostly the out-of-plane integrals of FC, this full cancellation does not take place, and one has a possibility of defining the FC through the measurements of flux tube probes, which is especially interesting for nonzero $T$, and around $T=T_{c}$ [56 58].

In the next section we define the connected flux tube probes via field correlators, following [43, 44] and adding a new contribution from the correlation $D_{1}^{(n p)}$.

\section{Flux tube fields via field correlators}

To measure field distributions around the static color charges $Q, \bar{Q}$, one can use the connected probe, defined by the contour $C$, shown in Fig.1, as it is done on the lattice [1-24], and calculated in the FCM, see e.g. [43, 44].

As shown in [44], Eq. (30), the resulting effective field $F_{\mu \nu}(x)$ is expressed via the FC, Eq. (1),

$$
F_{\mu \nu}(x)=\int_{S} d \sigma_{\alpha \beta}(y) g^{2} D_{\alpha \beta \mu \nu}^{(2)}(x-y) .
$$

Inserting (1) in (14) one obtains as in [44] the expression for the colorelectric probe $\mathbf{E}_{i}(\mathbf{r}, \mathbf{R})$

$$
E_{i}(\mathbf{r}, \mathbf{R})=n_{k} \int_{0}^{R} d l \int_{-\infty}^{\infty} d t\left(\delta_{i k} D^{E}(z)+\frac{1}{2} \frac{\partial\left(z_{i} D_{1}^{E}(z)\right)}{\partial z_{k}}\right),
$$

where $z=(\mathbf{r}-\mathbf{n} l, t)$, and $\mathbf{n}=\frac{\mathbf{R}}{R}$ is along the axis $x_{3}$, where the charges $Q, \bar{Q}$ are placed at the distance $R$, see Fig. 1 .

Inserting the perturbative part $D_{1}^{E(\text { pert) }}$ from (10), one obtains the screened color Coulomb field

$$
\mathbf{E}^{(1)}(\mathbf{r})=\frac{\left(N_{c}^{2}-1\right)}{2 N_{c}}\left(\frac{\alpha_{s} \mathbf{r} \chi(m r)}{r^{3}}-\frac{\alpha_{s}(\mathbf{r}-\mathbf{R})}{(\mathbf{r}-\mathbf{R})^{3}} \chi(m|\mathbf{r}-\mathbf{R}|)\right),
$$

where the screening factor is $\chi(z)=(1+z) e^{-z}$, and at the midpoint between the charges one has

$$
\mathbf{E}^{(1)}\left(\frac{\mathbf{R}}{2}\right)=\frac{4\left(N_{c}^{2}-1\right)}{N_{c}} \frac{\alpha_{s} \mathbf{R}}{R^{3}} \chi\left(\frac{m R}{2}\right) .
$$

Eq.(17) contains both the standard perturbative part $E^{(1)} \sim \frac{\alpha_{s}}{R^{2}}$, at $R \ll \frac{1}{m}$ and the nonperturbative screening $E^{(1)} \sim \frac{\alpha_{s} m}{R} e^{-m R}$ at $R \gg \frac{1}{m}$. For the field correlators, as in 
(14), the mass, $m \approx 1 \mathrm{GeV}$, while the screening in the OGE potential $V_{1}(r)$ Eq. (11), is much softer, as discussed in [59], see also appendix 2.

From (17) one can estimate $E_{3}^{(1)}$ at $R=0.2 \mathrm{fm}, m=1 \mathrm{GeV}$ and $r_{\perp}=0 . E_{3}^{(1)}(0.5 \mathrm{fm}, 0)=$ $0.122 \alpha_{s} \mathrm{GeV}^{2} \approx 0.05 \mathrm{GeV}^{2}$ for $\alpha_{s} \cong 0.4$. In a similar way, using the asymptotics (7), and the relation (3), one has

$$
D(z)=\frac{\sigma}{\pi \lambda^{2}} \exp \left(-\frac{|z|}{\lambda}\right), \quad \lambda^{-1} \cong 1 \mathrm{GeV},
$$

which yields for the colorelectric probe, following (15)

$$
\mathbf{E}^{D}=\mathbf{n} \frac{2 \sigma}{\pi} \int_{0}^{R / \lambda} d l\left|l \mathbf{n}-\frac{\mathbf{r}}{\lambda}\right| K_{1}\left(\left|l \mathbf{n}-\frac{\mathbf{r}}{\lambda}\right|\right) .
$$

For $R \rightarrow \infty$ one obtains from (19) the saturated colorelectric field at the distance $r_{\perp}$ from the axis

$$
E_{3}^{D}\left(\mathbf{r}_{\perp}\right)=2 \sigma\left(1+\frac{r_{\perp}}{\lambda}\right) \exp \left(-\frac{r_{\perp}}{\lambda}\right)
$$

and the saturated on-axis value $E_{3}^{\text {sat }}$ (on axis) $=2 \sigma$.

Summing up the contributions of (17), (19) for the field $E_{3}$ at the midpoint on the axis $\left(r_{\perp}=0\right)$, one has $\left(N_{c}=3\right)$

$$
E_{3}^{\mathrm{tot}}\left(\frac{R}{2}, r_{\perp}=0\right)=\frac{32 \alpha_{s}}{3 R^{2}} \chi\left(\frac{m R}{2}\right)+\frac{2 \sigma}{\pi} \int_{0}^{R / \lambda} d x \cdot x K_{1}(x) .
$$

Another interesting characteristics of flux tabes is the $E_{3}$ dependence on the distance to the $Q \bar{Q}$ axis, i.e. on $r_{\perp}$. Using (16), (19) one can write

$$
E_{3}\left(r_{\perp}\right) \equiv E_{3}\left(r_{\perp}, \frac{R}{2}\right)=E_{3}^{D}\left(r_{\perp}, \frac{R}{2}\right)+E_{3}^{(1)}\left(r_{\perp}, \frac{R}{2}\right)
$$

where

$$
E_{3}^{D}\left(r_{\perp}, \frac{R}{2}\right)=\frac{2 \sigma}{\pi} \int_{-\frac{R}{2 \lambda}}^{\frac{R}{2 \lambda}} d x \sqrt{x^{2}+\frac{r_{\perp}^{2}}{\lambda^{2}}} K_{1}\left(\sqrt{x^{2}+\frac{r_{\perp}^{2}}{\lambda^{2}}}\right),
$$

$E_{3}^{(1)}$ is given in (16),

$$
E_{3}^{(1)}\left(r_{\perp}, \frac{R}{2}\right)=\frac{4}{3} \alpha_{s} \frac{R \chi\left(m \sqrt{r_{\perp}^{2}+\frac{R^{2}}{4}}\right)}{\left(r_{\perp}^{2}+\frac{R^{2}}{4}\right)^{3 / 2}} .
$$

In the next section we compare our results for $E_{3}\left(r_{\perp}\right)$ and $E_{3}^{t o t}\left(\frac{R}{2}\right)$ with the lattice data [46].

We now turn to the effective magnetic monopole picture, which can be derived from our method, to compare it with the dual superconductor model.

To this end we as in [44] define first of all the magnetic current $\mathbf{k}$,

$$
\mathbf{k}=\operatorname{rot} \mathbf{E}(\mathbf{r}, \mathbf{R})=\operatorname{rot}\left(\mathbf{E}^{D}(\mathbf{r})+\mathbf{E}^{(1)}(\mathbf{r})\right) \equiv \mathbf{k}_{D}+\mathbf{k}^{(1)},
$$

One can deduce from (16), that $\mathbf{E}^{(1)}$ at $r_{3}=\frac{R}{2}$ (at the midpoint) does not have component along axis 1 and 2 , so that it can be written as $\mathbf{E}^{(1)}\left(r_{3}=\frac{R}{2}, r_{\perp}\right)=\mathbf{n} f^{(1)}\left(r_{\perp}^{2}\right)$, and hence $f^{(1)}\left(r_{\perp}^{2}\right)=E_{3}^{(1)}\left(r_{\perp}, \frac{R}{2}\right)$ given in (22) . 


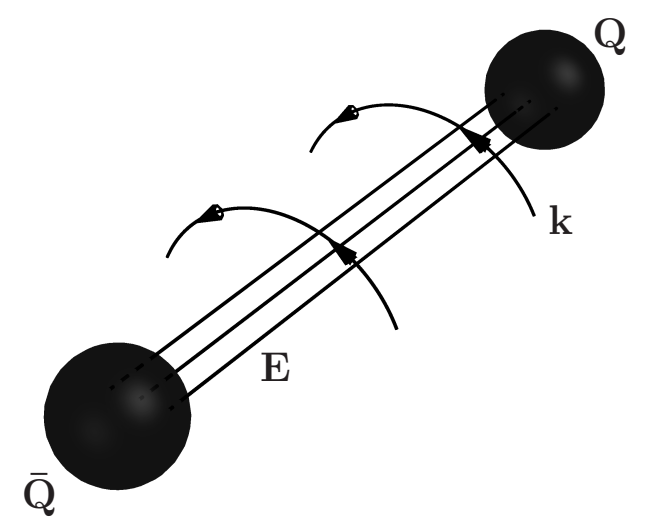

Figure 4: Colormagnetic current $k$ around the flux tube.

The same is true for $E^{D}$, Eq. (19), so that the total $\mathbf{k}=\mathbf{k}^{(1)}+\mathbf{k}_{D}$ is perpendicular to $\mathbf{n}$, as shown in Fig. 4 .

Therefore $k_{z}=0$, and $k_{x}=2 y f^{\prime}, k_{y}=-2 x f^{\prime}$ so that $\mathbf{k r}=0$, which means, that vectors $\mathbf{k}$ form circular loops around the $(Q, \bar{Q})$ axis. The function $f^{(1)}\left(r_{\perp}^{2}\right)=E_{3}^{(1)}$ is given in (24), and one can calculate the $r_{\perp}$ dependence $\left|\mathbf{k}_{\perp}^{(1)}\left(r_{\perp}\right)\right|^{2}=\left(k_{x}^{(1)}\left(r_{\perp}\right)\right)^{2}+\left(k_{y}^{(2)}\left(r_{\perp}\right)\right)^{2}$,

$$
\left(\mathbf{k}^{(1)}\left(r_{\perp}\right)\right)^{2}=4 r_{\perp}^{2}\left(\frac{\partial E_{3}^{(1)}}{\partial r_{\perp}^{2}}\right)^{2}
$$

The function $\mathbf{k}_{D}\left(r_{\perp}\right)$ is obtained from (23) $-z K_{0}(z)$, with the result

$$
\mathbf{k}_{D}^{2}\left(r_{\perp}\right)=\frac{4 \sigma^{2} r^{2}}{\pi^{2} \lambda^{4}}\left(\int_{-\frac{R}{2 \lambda}}^{\frac{R}{2 \lambda}} d x K_{0}\left(\sqrt{x^{2}+\frac{r_{\perp}^{2}}{\lambda^{2}}}\right)\right)^{2}
$$

In the case, when $\mathbf{k}_{D}$ and $\mathbf{k}^{(1)}$ can be both nonzero the resulting $\mathbf{k}\left(r_{\perp}\right)$ is

$$
\mathbf{k}\left(r_{\perp}\right)=\mathbf{k}^{(1)}+\mathbf{k}_{D}=-(\mathbf{n})_{\varphi} r_{\perp}\left(\frac{2 \sigma}{\pi \lambda^{2}} \int_{-\frac{R}{2 \lambda}}^{\frac{R}{2 \lambda}} d x K_{0}\left(\sqrt{x^{2}+\frac{r_{\perp}^{2}}{\lambda^{2}}}\right)+\frac{\partial f^{(1)}}{\partial r_{\perp}^{2}}\right)
$$

and $\mathbf{n}_{\varphi} \mathbf{r}_{\perp}=0, \mathbf{n}_{\varphi}^{2}=1$.

The most important point for the connection to the superconducting model is the dual London equation $\operatorname{rot} \mathbf{k}=\lambda^{-2} \mathbf{E}$, which, as shown in [44], is supported asymptotically $\left(r_{\perp} \rightarrow \infty\right)$ by the relation for the saturated string $(R \rightarrow \infty)$

$$
\operatorname{rot} \mathbf{k}_{D}=\gamma_{D}\left(r_{\perp}\right) \lambda^{-2} \mathbf{E}^{(D)}\left(r_{\perp}\right), \quad \gamma_{D}\left(r_{\perp}\right)=\frac{r_{\perp} / \lambda-2}{r_{\perp} / \lambda+1}, \gamma(\infty)=1 .
$$

\section{Results and discussion}

To compare with recent accurate lattice data [46], we are using the data, shown in Fig. 4 of this paper for two types of behavior: first, we are using the data [46] for $E_{3}^{\text {tot }}(R)$ here $R=0.76 \mathrm{fm}, 0.95 \mathrm{fm}, 1.14 \mathrm{fm}, 1.33 \mathrm{fm}$ and calculate our $E_{3}$ from Eq.(18) for these 
values of $R$. The results are shown in Fig. 5 with $\alpha_{s}=0.4, m=1 \mathrm{GeV}, \sigma=0.18 \mathrm{GeV}^{2}$. One can see a reasonable agreement of our theory with the data,where a slow decrease of $E_{3}^{\text {tot }}(R)$ is due to $E_{3}^{(1)}$, while the saturation at $E_{3}=2 \sigma$ is due to $E_{3}^{D}$.

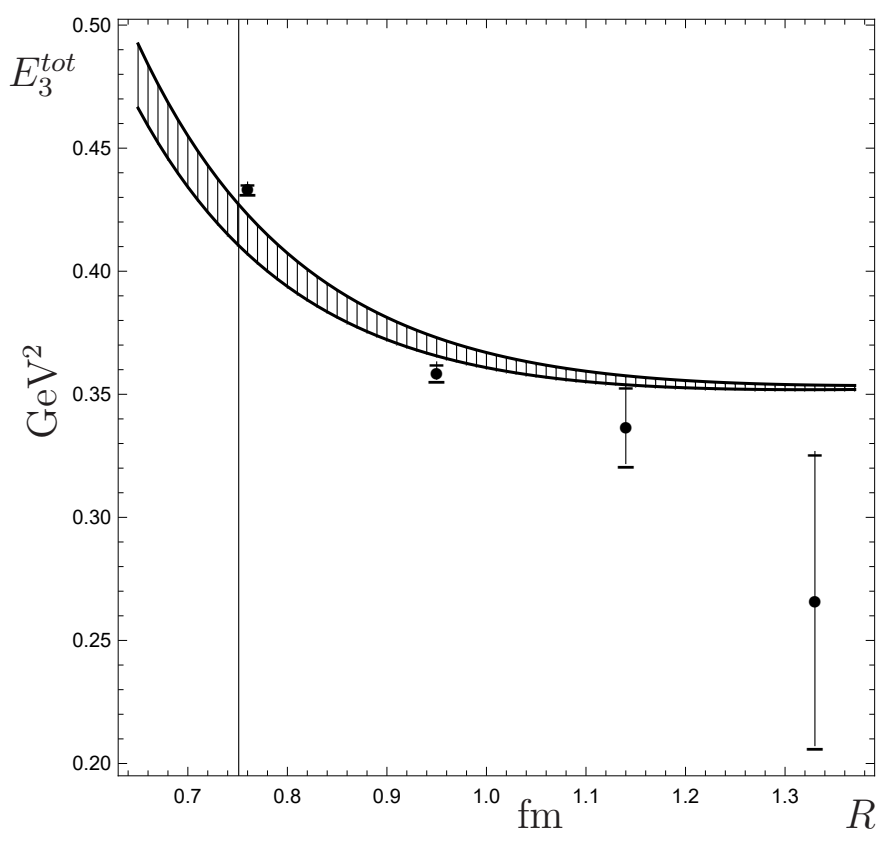

Figure 5: $E_{3}^{t o t}=E_{3}^{t o t}\left(\frac{R}{2}, r_{\perp}=0\right)$. The tube length dependence of the CE field strength at the midpoint. The shaded region corresponds to $\alpha_{s}=0.4$ (lower curve) and $\alpha_{s}=0.45$ (upper curve). The points with error bars are from the lattice measurements in [46].

To check the $r_{\perp}$ dependence, we again are using data of [46] and present our results for the quoted values of $R$ in Figs. 6-9. One can see again a reasonable agreement at the level of $O(5 \%)$ for $r_{\perp}<0.5 \mathrm{fm}$. Note, that our parameters $\alpha_{s}, m, \sigma$ are fixed at the physically relevant values, $\alpha_{s}(Q \sim 1 \mathrm{GeV})=0.4, \sigma=0.18 \mathrm{GeV}^{2}$.

Finally,in Fig. 10 we demonstrate the $r_{\perp}$ dependence of the modulus of $\left|\mathbf{k}\left(r_{\perp}\right)\right|$, given by Eqs. (26), (28). One can see the exponential decay at large $r_{\perp}$, typical for the color screening of massive gluon fields.

At this point one can compare our results with the MDS picture. In general, one can treat the dual abelian Higgs picture and different versions of MDS in the same language as in FC, calculating $D^{E}, D_{1}^{E}$ via solutions of Ginzburg-Landau equations etc.

This type of analysis was done in [59], where $D^{E}, D_{1}^{E}$ have been related with the dual filed propagator in the abelian Higgs model. However, parameters of the model and the form of $D, D_{1}$ are not fixed, e.g the connection of sigma and the mass $m=1 / \lambda$, whereas in the FC approach in QCD the product $\sigma \lambda^{2}$ is fixed by the gluelump mechanism and ensures Casimi scaling, observed on the lattice. Summarising, in the FC approach all observables are defined by the only nonperurbative scale (in addition to quark masses), which can be chosen as $\sigma$.

In [46] the theoretical form of $E_{3}\left(r_{\perp}\right)$ was chosen, according to the solutions of the Ginzburg-Landau equations, suggested in [60], with parameters, ensuring a good agreement with the lattice data. These parameters correspond to the superconductor of the first order, where the coherence length $\xi$ is larger, than the penetration length $\lambda$. However, the three flux tube parameters depend (moderately) on the length of the flux tube 


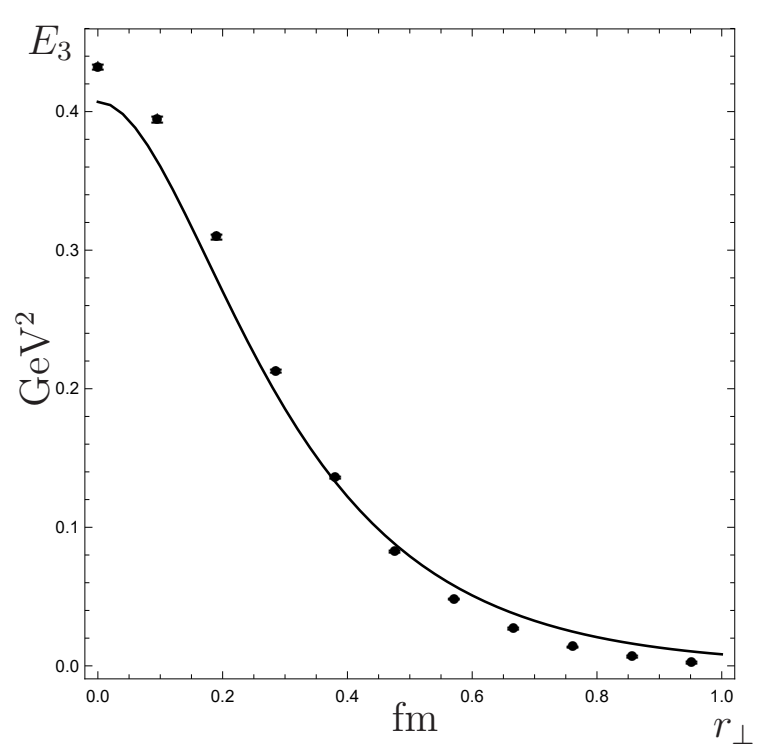

Figure 6: $E_{3}=E_{3}\left(r_{\perp}, R=0.76 \mathrm{fm}\right)$. The transverse radius dependence of the $\mathrm{CE}$ field strength for the fixed flux tube length $R=0.76 \mathrm{fm}$. The dots with error bars are from the lattice measurements in [46].

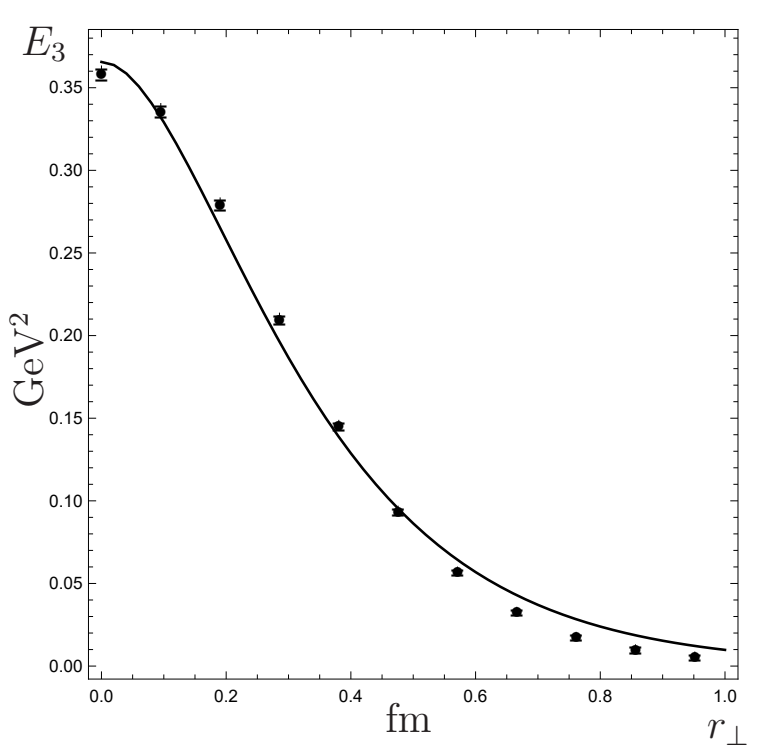

Figure 7: $E_{3}=E_{3}\left(r_{\perp}, R=0.95 \mathrm{fm}\right)$. The transverse radius dependence of the $\mathrm{CE}$ field strength for the fixed flux tube length $R=0.95 \mathrm{fm}$. The dots with error bars are from the lattice measurements in [46].

$R$.

Summarizing, we have derived two components of the CE fields in the flux tube and have shown the strong trasverse screening of CE fields on the length $\lambda=0.2 \mathrm{fm}$. We also found the slight decrease and saturation of the on-axis field $E_{3}(R)$ at large $R$. We have found a reasonably good agreement of our results with the latest lattice data of [46], confirming the applicablity of our theory using standard parameters, independent of $R$.

Finally, we have presented arguments, why the in-plane screening of the gluon exchange (color Coulomb interaction) is strongly damped, as compared with the transverse screening of the same interaction.

The authors are grateful to P. Cea, L. Cosmai, F. Cuteri and A. Papa for providing the numerical data.

This work was done in the framework of the scientific project, supported by the Russian Science Foundation grant \#16-12-10414.

\section{A Appendix: Calculation of the correlator $D_{1}$ via the gluelump Green's function}

Consider the field correlator Eq.(10), and take into account, that $F_{\mu \nu}=\partial_{\mu} A_{\nu}-\partial_{\nu} A_{\mu}-$ $i g\left[A_{\mu} A_{\nu}\right]$. The contribution of the first terms with derivatives immediately yields the lowest contribution in the form

$$
D_{1}^{E}(x)=-\frac{2 g^{2}}{N_{c}^{2}} \frac{d G(x)}{d x^{2}}
$$




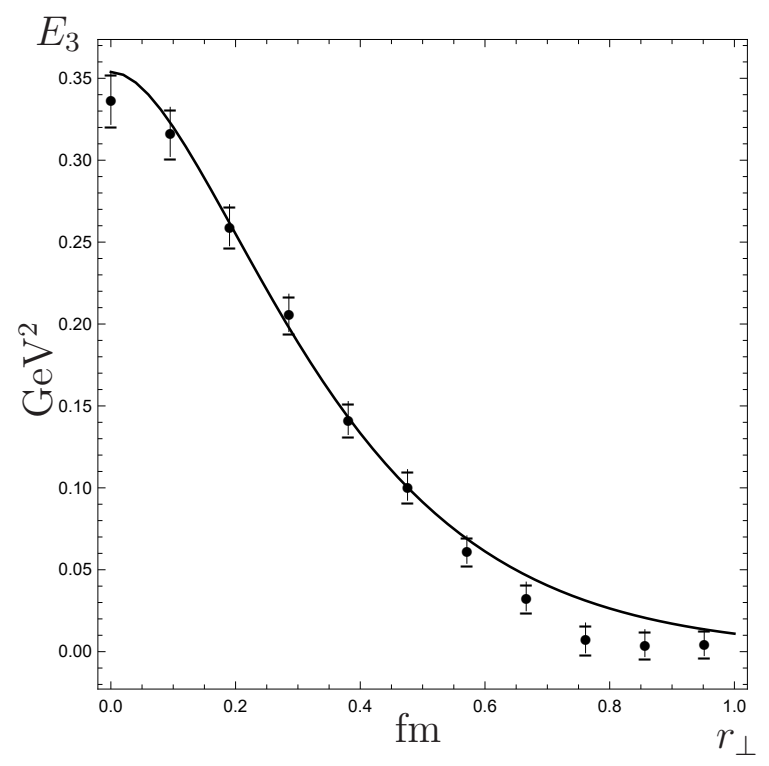

Figure 8: $E_{3}=E_{3}\left(r_{\perp}, R=1.14 \mathrm{fm}\right)$. The transverse radius dependence of the $\mathrm{CE}$ field strength for the fixed flux tube length $R=1.14 \mathrm{fm}$. The dots with error bars are from the lattice measurements in [46].

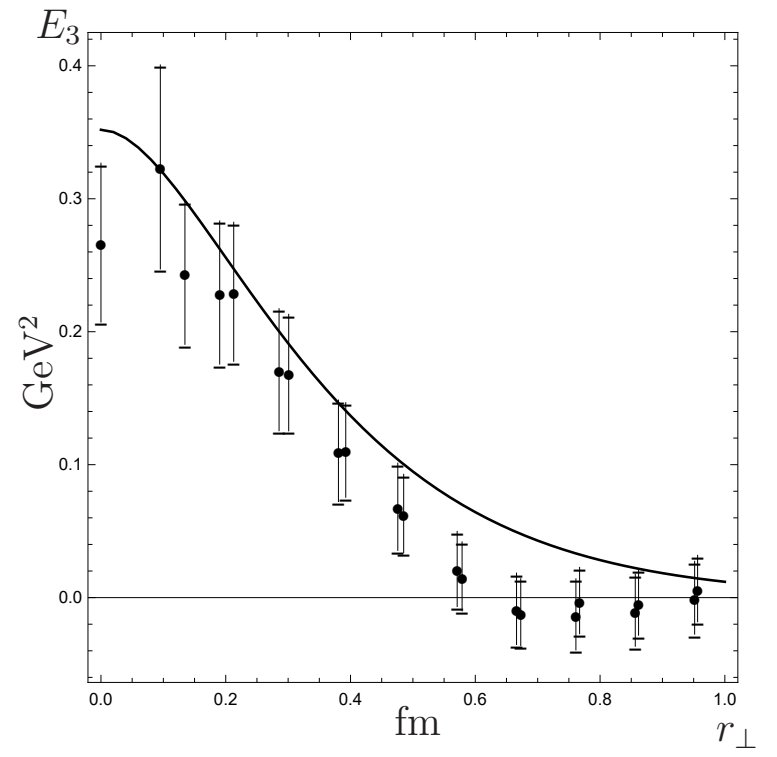

Figure 9: $E_{3}=E_{3}\left(r_{\perp}, R=1.33 \mathrm{fm}\right)$. The transverse raius dependence of the $\mathrm{CE}$ field strength for the fixed flux tube length $R=$ $1.33 \mathrm{fm}$. The dots with error bars are from the lattice measurements in [46].

where $G(x)$ is the one-gluon gluelump Green's function

$$
G_{\mu \nu}^{(1 g)}(x, y)=\left\langle\operatorname{Tr}_{a} A_{\mu}(x) \hat{\Phi}(x, y) A_{\nu}(y)\right\rangle=\delta_{\mu \nu} G(x-y)
$$

and $\hat{\Phi}(x, y)$ is the parallel transporter in the adjoint representation and we have exploited the Feynman gauge.

To simplify the matter we consider the gluelump Green's function as a relativistic Green's function of scalar particle with mass $m$ (neglecting internal degrees of freedom in the first approximation), which yields

$$
G(x)=\frac{\left(N_{c}^{2}-1\right) N_{c}}{4 \pi^{2}} \frac{m}{|x|} K_{1}(m|x|),
$$

where $K_{1}$ is the modified Bossel function. Taking derivative in (A.1), one has

$$
D_{1}^{E}(x)=\frac{g^{2} m^{2}}{4 \pi^{2}} \frac{\left(N_{c}^{2}-1\right)}{N_{c}} \frac{K_{2}(m|x|)}{x^{2}} .
$$

In the limit $m \rightarrow 0$ Eq. (A.4 yields the standard one-gluon form $D_{1}^{E}(x)=\frac{16 \alpha_{s}}{3 \pi x^{4}}$, which generates according to (5) the color Coulomb interaction $V_{1}(r)=-\frac{4 \alpha_{s}}{3 r}$.

In the paper the form (A.4) is used to predict the field distribution in the flux tube. 


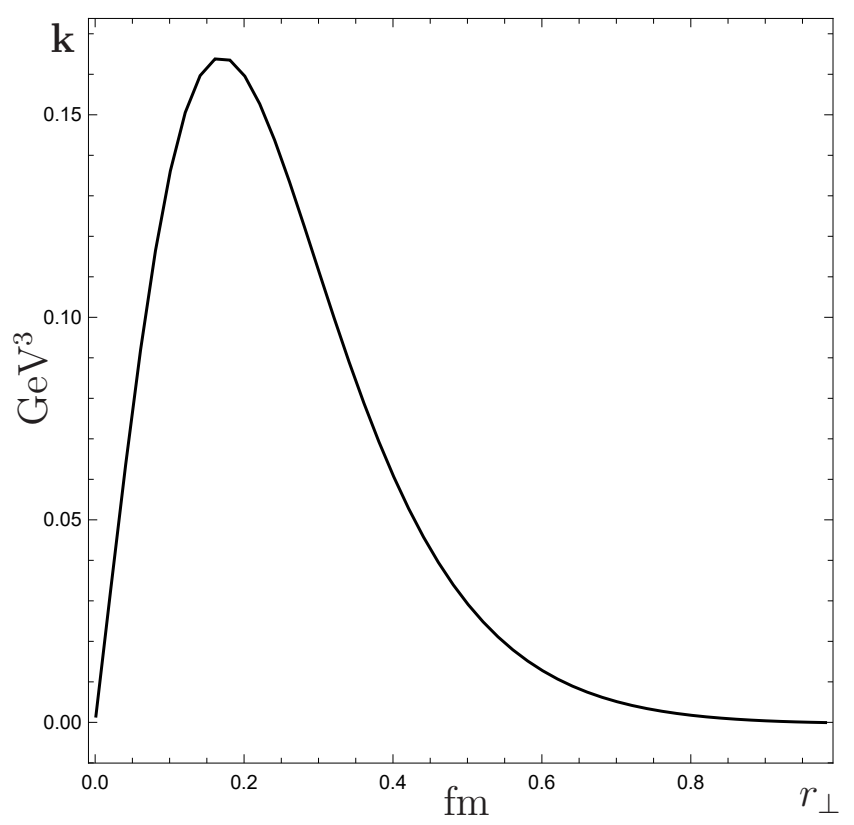

Figure 10: The transverse radius dependence of the CM current $\left|\mathbf{k}\left(r_{\perp}\right)\right|$ at $R=0.76 \mathrm{fm}$.

\section{References}

[1] M. Fukugita and T. Niuya, Phys. Lett. B 132, 374 (1983).

[2] J.E. Kiskis and K. Sparks, Phys. Rev. D 30, 1326 (1984).

[3] J.W. Flower and S.W. Otto, Phys. Lett. B 160, 128 (1985).

[4] J. Wosiek and R.W. Haymaker, Phys. Rev. D 36, 3297 (1987).

[5] A. Di Giacomo, M. Maggiore, and S. Olejnik, Phys. Lett. B 236, 199 (1990).

[6] A. Di Giacomo, M. Maggiore, and S. Olejnik, Nucl. Phys. B 347, 441 (1990).

[7] V. Singh, D.A. Browne, and R.W. Haymaker, Phys. Lett. B 306, 115 (1993) arXiv:hep-lat/9301004.

[8] P. Cea and L. Cosmai, Nucl. Phys. Proc. Suppl. 30, 572 (1993).

[9] Y. Matsubara, S. Ejiri, and T. Suzuki, Nucl. Phys. Proc. Suppl. 34, 176 (1994) arXiv:hep-lat/9311061].

[10] P. Cea and L. Cosmai, Nuovo Cim. A 107, 541 (1994) arXiv:hep-lat/9210030].

[11] P. Cea and L. Cosmai, Nucl. Phys. Proc. Suppl. 34, 219 (1994) arXiv:hep-lat/9311023.

[12] P. Cea and L. Cosmai, Phys. Lett. B 349, 343 (1995) arXiv:hep-lat/9404017].

[13] P. Cea and L. Cosmai, Nucl. Phys. Proc. Suppl. 42, 225 (1995) arXiv:hep-lat/9411048. 
[14] P. Cea and L. Cosmai, Phys. Rev. D 52, 5152 (1995) arXiv:hep-lat/9504008.

[15] G.S. Bali, K. Schilling, and C. Schlichter, Phys. Rev. D 51, 5165 (1995) arXiv:hep-lat/9409005.

[16] R.W. Haymaker and T. Matsuki, Phys. Rev. D75, 014501 (2007) arXiv:hep-lat/0505019.

[17] A. D'Alessandro, M. D’Elia, and L. Tagliacozzo, Nucl. Phys. B 774, 168 (2007) arXiv:hep-lat/0607014.

[18] M.S. Cardaci, P. Cea, L. Cosmai, R. Falcone, and A. Papa, Phys. Rev. D 83, 014502 (2011) arXiv:1011.5803 [hep-lat]].

[19] P. Cea, L. Cosmai, and A. Papa, Phys. Rev. D 86, 054501 (2012) arXiv:1208.1362 [hep-lat]].

[20] P. Cea, L. Cosmai, F. Cuteri, and A. Papa, in Proceedings, 31st International Symposium on Lattice Field Theory (Lattice 2013), PoS(LATTICE 2013)468 (2013) arXiv:1310.8423 [hep-lat]].

[21] P. Cea, L. Cosmai, F. Cuteri, and A. Papa, Phys. Rev. D 89, 094505 (2014) arXiv:1404.1172 [hep-lat]].

[22] P. Cea, L. Cosmai, F. Cuteri, and A. Papa, in Proceedings, 32nd International Symposium on Lattice Field Theory (Lattice 2014), PoS(LATTICE 2014)350 (2014) arXiv:1410.4394 [hep-lat]].

[23] N. Cardoso, M. Cardoso, and P. Bicudo, Phys. Rev. D 88, 054504 (2013) arXiv:1302.3633 [hep-lat]].

[24] M. Caselle, M. Panero, R. Pellegrini, and D. Vadacchino, JHEP 2015: 105 (2015) arXiv:1406.5127 [hep-lat]].

[25] G.'t Hooft, in: High Energy Physics, EPS International Conference, Palermo, 1975 (A. Zichichi, ed., 1975).

[26] S. Mandelstam, Phys. Rept. 23, 245 (1976).

[27] G. Ripka, Lect. Notes Phys. 639, 1 (2004).

[28] K.-I. Kondo, S. Kato, A. Shibata, and T. Shinohara, Phys. Rept. 579, 1 (2015) arXiv:1409.1599 [hep-th]].

[29] H.G. Dosch, Phys. Lett. B 190177 (1987).

[30] H.G. Dosch, Yu.A. Simonov, Phys. Lett. B 205339 (1988).

[31] Yu.A. Simonov, Nucl. Phys. B 307, 512 (1988).

[32] Yu.A. Simonov, in "QCD: Perturbative or Nonperturbative?" Proc. of the XVII Autumn School, Lisbon, Portugal, Eds. S. Ferreira, P. Nogueira, J.I. Silva-Marcos (Singapore: World Scientific, 2000).

[33] A.M. Badalian, Yu.A. Simonov and V.I. Shevchenko, Yad. Fiz. 69, 1808 (2006). 
[34] Yu.A. Simonov, Int. J. Mod. Phys. A 31, 1650016 (2016) arXiv:1509.06930 [hep$\mathrm{ph}]$.

[35] Yu.A. Simonov, Phys. At. Nucl. 58, 107 (1995) arXiv:hep-ph/9311247.

[36] A.V. Nefediev, Yu.A. Simonov and M.A. Trusov, Int. J. Mod. Phys. E 18, 549 (2009) arXiv:0902.0125 [hep-ph]].

[37] Yu.A. Simonov, Phys. Lett. B 619, 283 (2005) arXiv:hep-ph/0502078.

[38] I. Jorysz, C. Michael, Nucl. Phys. B 302, 448 (1988).

[39] N. Campbell, I. Jorysz, C. Michael, Phys. Lett. B 167, 91 (1986).

[40] Yu.A. Simonov, Phys. At. Nucl. 69, 528 (2006) arXiv:hep-ph/0501182.

[41] Yu.A. Simonov, V.I. Shevchenko, Adv. High Energy Phys. 2009, 873051 (2009) arXiv:0902.1405 [hep-ph]].

[42] Yu.A. Simonov, Proc. of the Steklov Inst. of Math. 272, 234 (2011) arXiv:1003.3608 [hep-ph]].

[43] Yu.A. Simonov, Phys. Usp. 39, 313 (1996), arXiv:hep-ph/9709344.

[44] D.S. Kuzmenko, V.I. Shevchenko, Yu.A. Simonov, Phys. Usp. 47, 3 (2004) arXiv:hep-ph/0310190].

[45] N.O. Agasian, M.S. Lukashov and Yu.A. Simonov, Eur. Phys. J. A 53: 138 (2017) arXiv:1701.07959 [hep-ph]].

[46] P. Cea, L. Cosmai, F. Cuteri, and A. Papa, arXiv:1702.06437 [hep-lat].

[47] P. Bicudo and V. Cardoso, Phys. Rev. D 85, 077501 (2012) arXiv:1111.1317 [heplat]].

[48] P. Bicudo, N. Cardoso, M. Cardoso, arXiv:1608.07742 [hep-lat].

[49] P. Cea, L. Cosmai, F. Cuteri, and A. Papa, JHEP 2016: 33 (2016) arXiv:1511.01783 [hep-lat]].

[50] Yu.A. Simonov, arXiv:1605.07060 [hep-ph].

[51] S. Gupta, K. Hübner, and O. Kaczmarek, Nucl. Phys. A 785, 278 (2007) arXiv:hep-lat/0608014.

[52] Yu.A. Simonov, Phys. At. Nucl. 68, 1294 (2005) arXiv:hep-ph/0406290.

[53] G.S. Bali, Phys. Rept. 343, 1 (2001) arXiv:hep-ph/0001312.

[54] T. Kawanai and S. Sasaki, Phys. Rev. Lett. 107, 091601 (2011) arXiv:1102.3246 [hep-lat]].

[55] K. Chung and J. Greensite, arXiv:1704.08995 [hep-lat].

[56] A.Di Giacomo, E. Meggiolaro, and H. Panagopoulos, Nucl. Phys. B 483371 (1997) arXiv:hep-lat/9603018. 
[57] M. D'Elia, A.Di Giacomo, and E. Meggiolaro, Phys. Rev. D 67, 114504 (2003) arXiv:hep-lat/0205018.

[58] A.Di Giacomo, H.G. Dosch, V.I. Shevchenko and Yu.A. Simonov, Phys. Rept. 372, 319 (2002) arXiv:hep-ph/0007223.

[59] M. Baker, N. Brambilla, H.G. Dosch and A. Vairo, Phys. Rev. D 58, 034010 (1998) arXiv:hep-ph/9802273.

[60] J.R. Clem, J. Low, Temp. Phys. 18, 427 (1975). 